\title{
Impurity states in $d$-wave superconductors with a competing antiferromagnetic interaction
}

\author{
Qiang Han, ${ }^{1,2}$ Tianlong Xia, ${ }^{2}$ Z. D. Wang,,${ }^{1,2, *}$ and X.-G. $\mathrm{Li}^{1}$ \\ ${ }^{1}$ Hefei National Laboratory for Physical Science at Microscale and \\ Department of Materials Science and Engineering, University of Science and Technology of China, Hefei, Anhui 230026, China \\ ${ }^{2}$ Department of Physics, University of Hong Kong, Pokfulam Road, Hong Kong, China
}

(Received 28 May 2003; revised manuscript received 15 December 2003; published 28 June 2004)

\begin{abstract}
Impurity states in $d$-wave superconductors with a competing antiferromagnetic (AF) order are investigated by solving the Bogoliubov-de Gennes equations. The potential scattering (PS) model with moderate strength and the Anderson impurity (AI) model with on-site hybridization are employed to describe the weak impurities. In zero external field, the impurity-induced AF order is rather weak and both models are able to give rise to impurity resonant states with close energy and similar profile of the local density of states. In the mixed state, the effect of magnetic-field-induced AF order on the impurity quasiparticle excitation is also examined. We find that the response of the impurity state to the presence of a local AF order is quite different for the two impurity models when a superconducting vortex is pinned by the impurity. For the PS model, the impurity resonance is subtly dependent on the sign and strength of the scattering potential, while for the AI model in the strong hybridization regime, the low-lying resonance is pinned near the Fermi level within the small gap opened by the AF order and is insensitive to the strength of the coupling between the impurity spin and the conduction electron. Based on our numerical results, we think that the two models give rise to different behaviors of the impurity resonances for both the nickel and zinc impurities in the magnetic field and the prospective scanning tunneling microscopic observation might give a clue to the dominant mechanism of the impurity states in the high- $T_{c}$ cuprates.
\end{abstract}

DOI: 10.1103/PhysRevB.69.224512

PACS number(s): 74.25.Jb, 72.15.Qm, 73.20.Hb

\section{INTRODUCTION}

The recent development of atomic-scale scanning tunneling microscopy (STM) has triggered much interest in the problem of impurity scattering in high- $T_{\mathrm{c}} d$-wave cuprates. A series of beautiful STM experiments have revealed local quasiparticle excitations induced by various defects, ${ }^{1}$ especially by impurity atoms (e.g., $\mathrm{Zn}$ and $\mathrm{Ni}$ ) directly doped into the $\mathrm{CuO}_{2}$ planes, ${ }^{2,3}$ which proved the ability of impurities as a powerful local probe in studying high- $T_{\mathrm{c}}$ superconductivity (HTS). However, there are still some controversies about the mechanism of the impurity resonant state and the modeling of the impurity interaction in HTS, especially when the impurity carries or induces magnetic moments as observed by the nuclear magnetic resonance ${ }^{4}$ (NMR) and neutron scattering $^{5}$ experiments. The potential scattering (PS) model $^{6-9}$ treats the impurity atom as a simple potential scatterer and, e.g., the $\delta$-function-type impurity interaction is

$$
H_{\mathrm{I}}^{\mathrm{PS}}=\left(V_{s}+V_{m}\right) c_{0 \uparrow}^{\dagger} c_{0 \uparrow}+\left(V_{s}-V_{m}\right) c_{0 \downarrow}^{\dagger} c_{0 \downarrow},
$$

where $c_{0 \sigma}^{\dagger}$ creates an electron of spin $\sigma$ at the site 0 containing the impurity. $V_{s}\left(V_{m}\right)$ denotes the strength of the scalar (magnetic) potential. According to the PS model, resonant states within the energy gap are produced by the scalar potential scatterers in a $d$-wave superconductor, which has been identified as one of the signatures of the $d$-wave pairing symmetry. In the PS model, the impurity spin is treated as a classical magnetic moment. On the other hand, different from the classical treatment of the impurity spin, several theoretical works ${ }^{10-13}$ attributed the origin of the impurity state to the quantum spin dynamics of impurity-related magnetic moments. In other words, the degrees of freedom of impurity spin should be included and interact with the conduction electrons. For instance, according to the Anderson impurity (AI) model, ${ }^{12,13}$ the internal degrees of freedom of the impurity are described by the Anderson $d$ electrons, and the impurity interaction is expressed in real space as

$$
H_{\mathrm{I}}^{\mathrm{AI}}=\sum_{\sigma} \epsilon_{d} d_{\sigma}^{\dagger} d_{\sigma}+U_{d} d_{\uparrow}^{\dagger} d_{\uparrow} d_{\downarrow}^{\dagger} d_{\downarrow}+t_{h} \sum_{\sigma}\left(c_{0 \sigma}^{\dagger} d_{\sigma}+\text { H.c. }\right),
$$

where $\epsilon_{d}$ is the $d$-electron energy level and $t_{h}$ is the strength of the hybridization of the moment with the conduction electron. The on-site hybridization between the $d$ level and the conduction electron as shown in Eq. (2) was found to be relevant to the $\mathrm{Ni}$ impurity, ${ }^{12}$ while the $\mathrm{Zn}$ impurity may be described by the four-site hybridization. ${ }^{13}$ In this paper, we simply accept the existence of the magnetic moments as an experimental fact and will not address their microscopic formation.

As will be seen later, both the PS model with a moderate strength of attractive scalar potential and the AI model with on-site hybridization are able to lead to the impurity quasiparticle resonance with quite similar quasiparticle spectra, consistent with one of the two impurity states ${ }^{14}$ associated with each Niatom observed by the STM experiment. ${ }^{3}$ To examine the dominant mechanism of the impurity state, in a previous work ${ }^{13}$ we studied the variation of the impurity resonance when a superconducting vortex with a normal metallic core was pinned right on the impurity site. However, the single LDOS peak associated with the vortex core in a normal metallic state of pure $d$-wave superconductors ${ }^{15}$ has never been observed experimentally. As studied theoretically for HTS with strong electron correlation, competing orderings such as the antiferromagnetic (AF) (Refs. 16-20) order 
and the staggered flux ${ }^{21,22}$ order might nucleate around the vortex core where the superconducting order is suppressed and thus a subgap opens at the vortex core, as observed by STM experiments. ${ }^{23}$ Recent experiments ${ }^{24-26}$ have indicated the existence of AF order inside the vortex in various cuprates. Here, we will examine the response of the impurity state to the presence of the static AF order. We find that in the PS model, the impurity state is produced at the vicinity of the vortex core state and the resonance energy is sensitively dependent on the sign and magnitude of the scattering potential. On the other hand, in the AI model the lowest-lying impurity resonance is generated with the spin polarization contrary to that of the vortex center. For both models, the impurity states lose the particle-hole dualism due to the destruction of the superconducting order (by the AF order) in the vortex core.

The rest of the paper is organized as follows. In Sec. II, we describe the effective Hubbard model to study the $d$-wave superconductivity with a competing AF order. In Secs. III and IV, the impurity states in both the PS model and the AI model are studied in zero field and in the mixed state. We give a brief summary in Sec. V.

\section{MODEL AND BASIC EQUATIONS}

In this work, we adopt a well-established $t-U-V$ Hubbard model $^{8}$ on a two-dimensional (2D) lattice with the on-site repulsion $(U)$ and the nearest-neighbor $(\mathrm{NN})$ pairing interaction $(V)$ to model the competing $\mathrm{AF}$ order and the $d$-wave superconducting (DSC) order, respectively. The model Hamiltonian is expressed as

$$
\begin{aligned}
H_{\mathrm{dsc}}= & -\sum_{\langle i, j\rangle \sigma} t_{i j} c_{i \sigma}^{\dagger} c_{j \sigma}+\sum_{\langle i, j\rangle \sigma}\left(\Delta_{i, j} c_{i \uparrow}^{\dagger} c_{j \downarrow}^{\dagger}+\text { H.c. }\right) \\
& +\sum_{i, \sigma}\left(U n_{i \bar{\sigma}}-\mu\right) c_{i \sigma}^{\dagger} c_{i \sigma},
\end{aligned}
$$

where $c_{i \sigma}^{\dagger}$ creates an electron of spin $\sigma$ at the $i$ th site. $n_{i \sigma}$ $=\left\langle c_{i \sigma}^{\dagger} c_{i \sigma}\right\rangle$ is the electron density with spin $\sigma$. In an external magnetic field, the hopping integral can be written as $t_{i j}$ $=t \exp \left[i \phi\left(\mathbf{r}_{\mathbf{i}}, \mathbf{r}_{\mathbf{j}}\right)\right]$ with $\phi\left(\mathbf{r}_{\mathbf{i}}, \mathbf{r}_{\mathbf{j}}\right)=-i\left(\pi / \Phi_{0}\right) \int_{\mathbf{r}_{\mathbf{i}}}^{\mathbf{r}_{\mathbf{j}}} \mathbf{A}(\mathbf{r}) \cdot d \mathbf{r}$ for the $\mathrm{NN}$ sites $\langle i, j\rangle, \mathbf{A}(\mathbf{r})$ the vector potential, and $\Phi_{0}=h c / 2 e$ the superconducting flux quantum. The internal field induced by supercurrents around the vortex core is neglected since high$T_{c}$ cuprates can be treated as extreme type-II superconductors. Therefore, $\mathbf{A}(\mathbf{r})$ is approximated as $(0, B x, 0)$ in a Landau gauge, where $B$ is the uniformly applied external magnetic field. $\mu$ is the chemical potential. $\Delta_{i, j}$ is the bond paring potential defined as $\Delta_{i j}=(V / 2)\left(\left\langle c_{i \uparrow} c_{j \downarrow}\right\rangle-\left\langle c_{i \downarrow} c_{j \uparrow}\right\rangle\right)$.

In the treatment of the AI model at low temperatures, $U_{d}$ in Eq. (2) is assumed to be infinite as usual, which forbids double occupancy of electrons on the $d$ level. Therefore, the slave-boson mean-field theory ${ }^{27}$ can be applied as in Refs. 11-13, where the $d$-electron operator is written as $d_{\sigma}^{\dagger}=f_{\sigma}^{\dagger} b$ with $f_{\sigma}$ the spin-carrying fermion operator and $b$ the holon operator. Furthermore, the single occupancy constraint $\Sigma_{\sigma} f_{\sigma}^{\dagger} f_{\sigma}+b^{\dagger} b=1$ should be obeyed. At the mean-field level, the holon operators $b$ and $b^{\dagger}$ are approximated by a $c$-number $b_{0}$ and the constraint is enforced on average by introducing a
Lagrange multiplier $\lambda_{0}$. Accordingly, the mean-field $H_{\mathrm{I}}^{\mathrm{AI}}$ becomes $H_{\mathrm{I}}^{\mathrm{AI}}=\Sigma_{\sigma} \tilde{\epsilon}_{d} f_{\sigma}^{\dagger} f_{\sigma}+\tilde{t}_{h} \Sigma_{\sigma}\left(c_{0 \sigma}^{\dagger} f_{\sigma}+\right.$ H.c. $)+\lambda_{0}\left(b_{0}^{2}-1\right)$ with renormalized parameters $\tilde{\epsilon}_{d}=\epsilon_{d}+\lambda_{0}$ and $\tilde{t}_{h}=t_{h} b_{0}$.

Based on the self-consistent mean-field approximation and the Bogoliubov transformation, diagonalization of the Hamiltonian $H_{\mathrm{dsc}}+H_{\mathrm{I}}$ can be achieved by solving the following Bogoliubov-de Gennes (BdG) equations:

$$
\sum_{j}\left(\begin{array}{cc}
H_{i j, \sigma} & \Delta_{i, j} \\
\Delta_{i, j}^{*} & -H_{i j, \bar{\sigma}}^{*}
\end{array}\right)\left(\begin{array}{c}
u_{j \sigma}^{n} \\
v_{j \bar{\sigma}}^{n}
\end{array}\right)=E_{n}\left(\begin{array}{c}
u_{i \sigma}^{n} \\
v_{i \bar{\sigma}}^{n}
\end{array}\right),
$$

where $u^{n}, v^{n}$ are the Bogoliubov quasiparticle amplitudes with corresponding eigenvalue $E_{n}$. In the PS model, $i, j$ represents the index of the 2D lattice site. $H_{i j, \sigma}=-\delta\left(\mathbf{r}_{i}+\boldsymbol{\tau}\right.$ $\left.-\mathbf{r}_{j}\right) t_{i, j}+\delta\left(\mathbf{r}_{i}-\mathbf{r}_{j}\right)\left[U n_{i \bar{\sigma}}-\mu+\delta\left(\mathbf{r}_{i}-\mathbf{r}_{0}\right) V_{s}\right]$ with $\boldsymbol{\tau}$ the unit vectors $\pm \hat{\mathbf{e}}_{x}$ and $\pm \hat{\mathbf{e}}_{y} . n_{i \sigma}$ is subject to the self-consistent conditions

$$
\begin{gathered}
n_{i \uparrow}=\sum_{n}\left|u_{i \uparrow}^{n}\right|^{2} f\left(E_{n}\right), \\
n_{i \downarrow}=\sum_{n}\left|v_{i \downarrow}^{n}\right|^{2}\left[1-f\left(E_{n}\right)\right],
\end{gathered}
$$

where $f(E)=\left[\exp \left(E / k_{B} T\right)+1\right]^{-1}$ represents the Fermi distribution function. In terms of $n_{i \sigma}$, the staggered magnetization of the AF order parameter is defined by

$$
M_{s}=(-)^{i}\left(n_{i \uparrow}-n_{i \downarrow}\right) \text {. }
$$

Here, the pairing potential $\Delta_{i, j}$ is defined between a pair of NN sites on the 2D lattice and is calculated self-consistently,

$$
\Delta\left(\mathbf{r}_{\mathbf{i}}, \mathbf{r}_{\mathbf{j}}\right) \equiv \Delta_{i, j}=\frac{V}{4} \sum_{n}\left(u_{i \uparrow}^{n} v_{j \downarrow}^{n^{*}}+u_{j \uparrow}^{n} \vartheta_{i \downarrow}^{n^{*}}\right) \tanh \left(\frac{E_{n}}{2 k_{B} T}\right),
$$

and the gauge-invariant $d$-wave order parameter $\Delta_{d}$ is defined as

$$
\Delta_{d}\left(\mathbf{r}_{\mathbf{i}}\right)=\frac{1}{4} \sum_{\tau} \Delta\left(\mathbf{r}_{\mathbf{i}}, \mathbf{r}_{\mathbf{i}}+\tau\right) e^{i \phi\left(\mathbf{r}_{\mathbf{i}}, \mathbf{r}_{\mathbf{i}}+\tau\right)} e^{2 i \theta(\tau)},
$$

where $\theta(\tau)=0, \pi / 2, \pi, 3 \pi / 2$ for $\boldsymbol{\tau}=\hat{\mathbf{e}}_{x}, \hat{\mathbf{e}}_{y},-\hat{\mathbf{e}}_{x}$, and $-\hat{\mathbf{e}}_{y}$.

In the AI model, $i, j$ in Eq. (4) should include an extra index $i_{d}$ representing the degree of freedom of the $d$ level in addition to the $2 \mathrm{D}$ lattice. There are hopping matrix elements between the index of the 2D lattice $i_{L}$ and $i_{d}$, namely $H_{i_{L} i_{d}, \sigma}=H_{i_{d} i_{L}, \sigma}=\delta\left(\mathbf{r}_{i}-\mathbf{r}_{0}\right) \tilde{t}_{h}$. And furthermore $H_{i_{d}{ }^{i} d}, \sigma=\widetilde{\epsilon}_{d}$. The expression of the bosonic number is

$$
b_{0}^{2}=1-\sum_{n}\left\{\left|u_{i_{d}{ }^{\prime}}^{n}\right|^{2} f\left(E_{n}\right)+\left|v_{i_{d} \downarrow}^{n}\right|^{2}\left[1-f\left(E_{n}\right)\right]\right\} .
$$

With the help of the derived equations (4)-(10), we can perform the self-consistent calculation as follows. For a given initial distribution of various order parameters, i.e., $n_{i \sigma}$ and $\Delta_{i, j}$, the BdG equations are numerically diagonalized and the obtained quasiparticle spectrum is used to calculate the new order parameters for the next iteration step. The results are obtained when expected accuracy is achieved with the relative errors of $n_{i \sigma}$ and $\Delta_{i, j}$ between two consecutive iteration steps being $\leqslant 0.1 \%$. Furthermore, for the AI model, the 
(a)

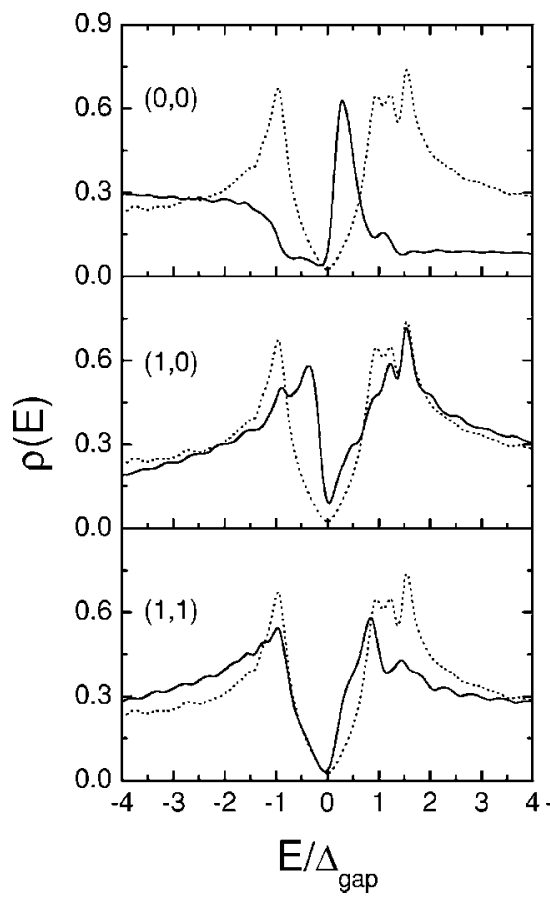

(b)

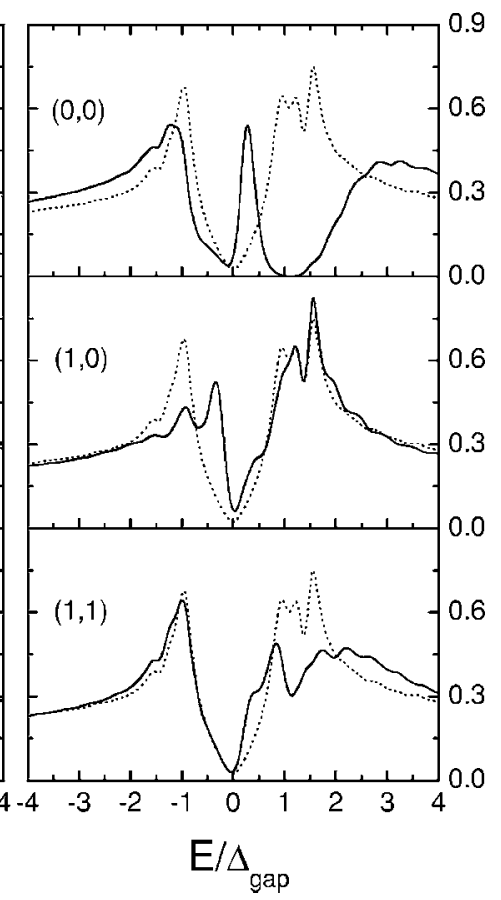

FIG. 1. The local density of states as a function of energy at the impurity site $(0,0), \mathrm{NN}$ site $(1,0)$, and NNN site $(1,1)$ in the PS model with $V_{s}=-3$ (a) and in the AI model with $\epsilon_{d}=-0.5$ and $t_{h}=1.0(\mathrm{~b})$, respectively. The dotted lines represents the local density of states at $(16,16)$, i.e., on the boundary of the $32 \times 32$ subsystem. The thermal broadening temperature is 0.02 .
Lagrange multiplier $\lambda_{0}$ is determined by minimizing the free energy of the system and $b_{0}$ is self-consistently calculated according to Eq. (10). Once the self-consistent quasiparticle spectrum is obtained, we calculate the LDOS,

$$
\rho\left(\mathbf{r}_{i}, E\right)=-\sum_{n}\left[\left|u_{i \uparrow}^{n}\right|^{2} f^{\prime}\left(E_{n}-E\right)+\left|v_{i \downarrow}^{n}\right|^{2} f^{\prime}\left(E_{n}+E\right)\right],
$$

which is proportional to the differential tunneling conductance observed in STM experiments.

In the following sections, the energy and length will be measured in units of the hopping integral $t$ and the lattice constant $a$.

\section{IMPURITY STATES WITHOUT MAGNETIC FIELD}

In this section, we study the impurity states without applying the magnetic field. To make the exact diagonalization of the BdG equations feasible and achieve the desired resolution to resolve the impurity resonance in energy, we address the system made of a periodic array $\left(M_{x} \times M_{y}\right)$ of subsystems each with the size $N_{x} \times N_{y}$ in the numerical calculation. We then define the Bloch states labeled by a Bloch momentum $\mathbf{k}=\left(2 \pi m_{x} / M_{x} N_{x}\right) \hat{\mathbf{e}}_{x}+\left(2 \pi m_{y} / M_{y} N_{y}\right) \hat{\mathbf{e}}_{y}$ with $m_{x, y}=0,1, \ldots, M_{x, y}-1$ and then the Hamiltonian of the total system is block-diagonalized for each $\mathbf{k}$. Therefore, the eigenvalue problem of the whole system with size $M_{x} N_{x}$ $\times M_{y} N_{y}$ reduces to diagonalize the subsystem of smaller size $N_{x} \times N_{y}$ for each $\mathbf{k}$. For each subsystem, the periodic boundary condition is applied. This method is well established and has been applied to study the effect of various inhomogeneities such as impurities and surfaces in $d$-wave superconductors. In our study, the total system consists of $10 \times 10$ subsystems each with the size $32 \times 32$.
As a model calculation, we choose the pairing interaction $V=1.2$ and the on-site repulsion $U=2.4$ such that the $d$-wave superconducting state overwhelms the AF order at the optimal doping $x=0.15$ (in other words, the average electron number per site $\bar{n}=0.85$ ) in the absence of magnetic field. At near-zero temperature where our calculations are performed, the above parameters give rise to $\Delta_{d} \simeq 0.09$ in zero field and accordingly we find that $\Delta_{\text {gap }} \simeq 0.36$, with $\pm \Delta_{\text {gap }}$ denoting the energy positions where the coherence peaks of the LDOS curve are located, as shown in Fig. $1 .^{28}$ Figures 1(a) and 1(b) plot the local density of states as a function of energy around the impurity site in the PS model with a negative (attractive) scattering potential $V_{s}=-3$ and the AI model with $\epsilon_{d}$ $=-0.5, t_{h}=1.0$ (strong hybridization regime), respectively. As shown in the figure, both models give rise to impurity resonance states with very close energy value $\omega / \Delta_{\text {gap }}=0.3$ and the impurity quasiparticle excitation has both electron and hole components. The electron part $\left|u_{i}(\omega)\right|^{2}$, which corresponds to an LDOS peak at $\omega$ in Fig. 1, has the largest amplitude right on the impurity site and decays rapidly with the distance from the center. On the other hand, the hole part $\left|v_{i}(\omega)\right|^{2}$ leading to an LDOS peak at $-\omega$ has vanishing amplitude on the impurity and the NNN site with its largest value on the NN site of the impurity. The resonance energy and the spatial distribution of the quasiparticle resonant state are consistent with the STM experiment ${ }^{3}$ of Ni-doped $\mathrm{Bi}_{2} \mathrm{Sr}_{2} \mathrm{CaCu}_{2} \mathrm{O}_{8+\delta}$. Our numerical results are also consistent with the non-self-consistent Green's-function analysis of the AI model in the continuum model. ${ }^{12}$ According to our selfconsistent study, in both models the coherence peaks at $\pm \Delta_{\text {gap }}$ are reduced at the vicinity of the impurity site due to the suppression of the DSC order. On the other hand, the LDOS at the impurity site is largely altered within the whole energy range in the PS model, while in the AI model the LDOS is 
hardly changed in the energy axis opposite to the resonance energy $\omega$, indicating that the spectra of the AI model agree with the STM observation better. Similar results can also be seen from the LDOS of the NN and NNN sites of the impurity in Fig. 1.

\section{IMPURITY STATES IN THE MIXED STATE}

In the presence of a strong magnetic field, the superconductor is in the mixed state. A magnetic unit cell which accommodates two vortices is selected as the subsystem with linear dimensions satisfying $N_{x} N_{y}=2 \Phi_{0} / B$ according to the theory of the magnetic translation group. Similar to the problem studied in the absence of a magnetic field, we are able to numerically handle the smaller subsystem for each of the magnetic Bloch quasimomenta $\mathbf{k}$. Note that in agreement with the symmetry of the magnetic translation group, the modified periodic boundary condition ${ }^{29}$ is applied and accordingly $\Delta_{d}\left(\mathbf{r}_{i}\right)$ satisfies the following translation relation: $\quad \Delta_{d}\left(\mathbf{r}_{i}+\mathbf{R}\right)=\Delta_{d}\left(\mathbf{r}_{i}\right) \exp \left[i \chi\left(\mathbf{r}_{i}, \mathbf{R}\right)\right]$, where $\chi\left(\mathbf{r}_{i}, \mathbf{R}\right)$ $=\left(2 \pi / \Phi_{0}\right) \mathbf{A}(\mathbf{R}) \cdot \mathbf{r}_{i}+4 \pi m n$ and $\mathbf{R}=m N_{x} \hat{\mathbf{e}}_{x}+n N_{y} \hat{\mathbf{e}}_{y}$ is the magnetic translation vector. Here a $10 \times 10$ array of the magnetic unit cell is chosen with the size of each unit cell $20 \times 40$.

Before studying the impurity state in the vortex center, for comparison we need first to study the electronic structure of the impurity-free vortex lattice of $d$-wave superconductors. It was first shown in Ref. 17 that the LDOS at the vortex core exhibited a double-peak structure near the Fermi level due to the local AF order induced near the vortex core where DSC order was suppressed, and later such an issue was intensively studied and similar results reproduced by many authors. ${ }^{18,19}$ Here we adopt a configuration of the AF order in which the $\mathrm{AF}$ order alternates sign from one vortex to its $\mathrm{NN}$ vortex along both the $\hat{\mathbf{e}}_{x}$ and $\hat{\mathbf{e}}_{y}$ directions as shown in Fig. 2(a), which plots the pattern of the staggered magnetization of the local AF order as defined in Eq. (7). This configuration is different from that in Ref. 17, where the AF order only changes sign along one of two directions, and that in Refs. 18 and 19, where the AF order has the same periodicity as the vortex lattice. Although the configuration we take has slightly higher free energy than that reported in Ref. 19 for higher magnetic field (i.e., smaller magnetic unit cell), our configuration ensures that the $\mathrm{AF}$ order parameter vanishes at the border of the vortex unit cell where DSC order recovers and might come closer to the most favorable configuration in low field as in the STM experiments. Despite this, the impurity state in the vortex center is mainly related to the localized nature of the AF core and is therefore insensitive to the detailed configurations of the AF order. Figure 2(b) displays the LDOS at the vortex core together with that at the midpoint between two NN vortices in full agreement with previous works. ${ }^{18,19}$ It can be seen that the AF order induced around the vortex core splits the zero bias peak (ZBP) (Ref. $15)$ of the LDOS predicted for a pure $d$-wave superconductor with normal metallic core due to the lifting of the spin degeneracy by the AF order. The AF order opens a small effective gap with gap edges denoted as $A$ and $B$ in Fig. 2(b). The LDOS peaks $A$ and $B$ come from the contributions of vortex core states with spin-up and spin-down polarization, (a)
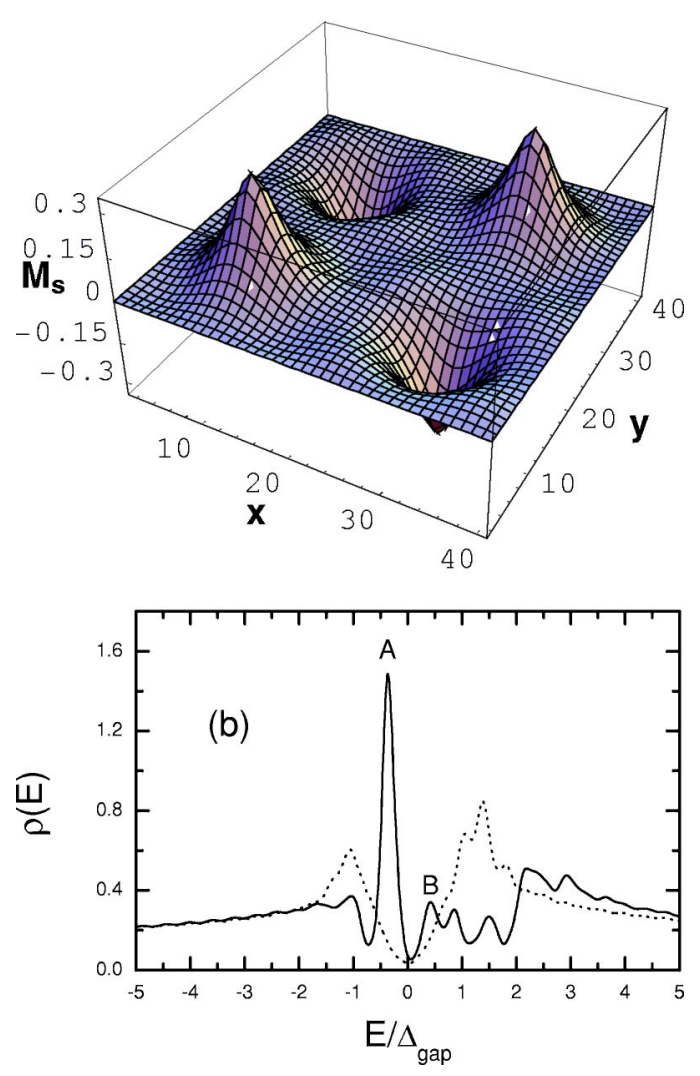

FIG. 2. (a) The spatial distribution of the staggered magnetization $M_{s}$ in four adjacent vortex unit cells with total size $40 \times 40$. (b) The LDOS at the vortex core (solid line) and at the site midway between two NN vortices (dotted line). The thermal broadening temperature is 0.02 .

respectively. Small peaks outside of the subgap $E_{A B}$ are the suppressed DSC coherence peaks and peaks corresponding to the von Hove singularity.

Next we study the impurity state at the vortex core in the presence of AF order in the PS model. Due to the presence of impurity scattering, both the staggered magnetization of the $\mathrm{AF}$ order $M_{s}$ and the electron number $n_{i}=n_{i \uparrow}+n_{i \downarrow}$ are varied around the impurity site. In Fig. 3, the variation of $M_{s}$ and $n$ is displayed along the $(1,0)$ direction across the vortex core (impurity site) at $(11,11)$ for a set of values of $V_{s}$. According to Fig. 3, the effect of the potential scattering on $M_{s}$ extends a few lattice sites while that on $n$ is quite local. From Fig. 3(a), except for the weak potential $V_{s}=-1$, the potential scattering tends to reduce the magnetization right on the impurity site regardless of the sign of the potential, and more interestingly we observe a net spin $S_{z}=\frac{1}{2}$ by summing the local moments induced around the impurity. Combining with the result in the zero-field case as reported recently in Ref. 30, we find that the magnetic field also plays an important role in the local moment formation in addition to $V_{s}$ and $U$. Moreover, we find from Fig. 3(b) that the sign of the variation of $n$ due to the potential scattering is always opposite to $V_{s}$, which is consistent with the fact that the negative (positive) potential attracts (repels) electrons to (from) it. Because of 


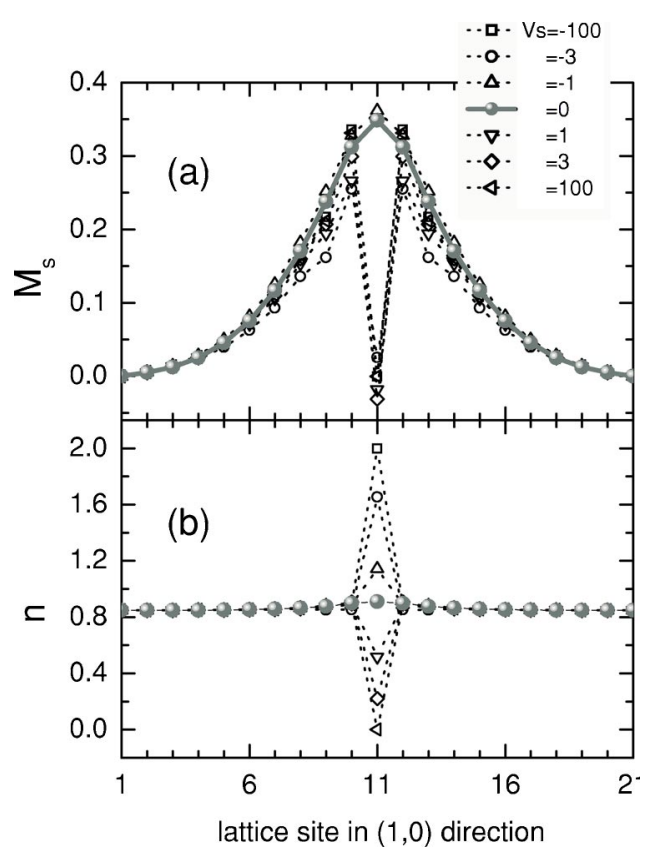

FIG. 3. The magnetization $M_{s}$ (a) and electron number $n$ (b) as a function of lattice site in the $(1,0)$ direction across the vortex core.

the self-consistent nature of the BdG equation, the impurityinduced variations of $M_{s}$ and $n$ (in other words $n_{i \uparrow}$ and $n_{i \downarrow}$ ), i.e., $\delta M_{s}=M_{s}\left(V_{s}\right)-M_{s}(0)$ and $\delta n=n\left(V_{s}\right)-n(0)$, are reflected in the mean-field Hamiltonian (3), resulting in the original pointlike nonmagnetic impurity acting as an extended classical magnetic scatterer with an effective unscreened magnetic moment $1 / 2$ when the potential scattering is large enough
$\left(V_{s}\right.$ exceeds a critical value $\left.{ }^{30}\right)$. Therefore, even though the scattering potential in the PS model might be pointlike and scalar itself, its overall effect is extended and magnetic within the vortex centers with the induced AF order. According to Fig. 3, the impurity-induced variation of $M_{s}$ and $n$ is short-ranged within a few lattice sites of the scatterer and the main contribution comes from the scattering center, so the total impurity effect can be treated simply as a point magnetic potential scattering with $\widetilde{V}_{s}=V_{s}+U \delta n_{0} / 2$ and $\widetilde{V}_{m}$ $=-U \delta M_{s 0} / 2$, where $\delta n_{0}$ and $\delta M_{s 0}$ denote the value of $\delta n$ and $\delta M_{s}$ at the impurity site, respectively. From Fig. 3, we have $\widetilde{V}_{s} \approx-98.8,-2.0,-0.76,0.52,2.2,98.8$ and $\widetilde{V}_{m} \approx-0.4$ (except for $\widetilde{V}_{m} \approx 0.0$ for $V_{s}=-1$ ) for $V_{s}=-100,-3,-1,1,3,100$ and $U=2.4$. The magnitude of $\tilde{V}_{s}$ is less than that of $V_{s}$ since the sign of $\delta n$ is always opposite to $V_{s}$. And for the parameters we studied, $\widetilde{V}_{m}$ is negligibly weak compared to the relatively large scattering potential $\left|V_{s}\right| \gtrsim 3$ while important for weak $V_{s}$ at the vicinity of 1 .

The resulting LDOS at the impurity site in the PS model is shown in Fig. 4 with various strengths of the scattering potential. Inspection of the impurity-induced states shows that they have close relevancy with the AF vortex core states [peaks $A$ and $B$ in Fig. 2(b)]. Here we pay attention to the low-lying impurity states within the subgap $E_{A B} .{ }^{31}$ From the figure, we find that in each case the appearance of the impurity state and the suppression of the vortex core states happen together at the impurity site. For the negative potential, there is an impurity resonance peak at the left side of the vortex state peak $A$. The low-lying LDOS peak within the subgap $E_{A B}$ is located at the right side of peak $A$ for $V_{s}=-3$ and at the left side of peak $B$ for $V_{s}=-1$. For the larger positive

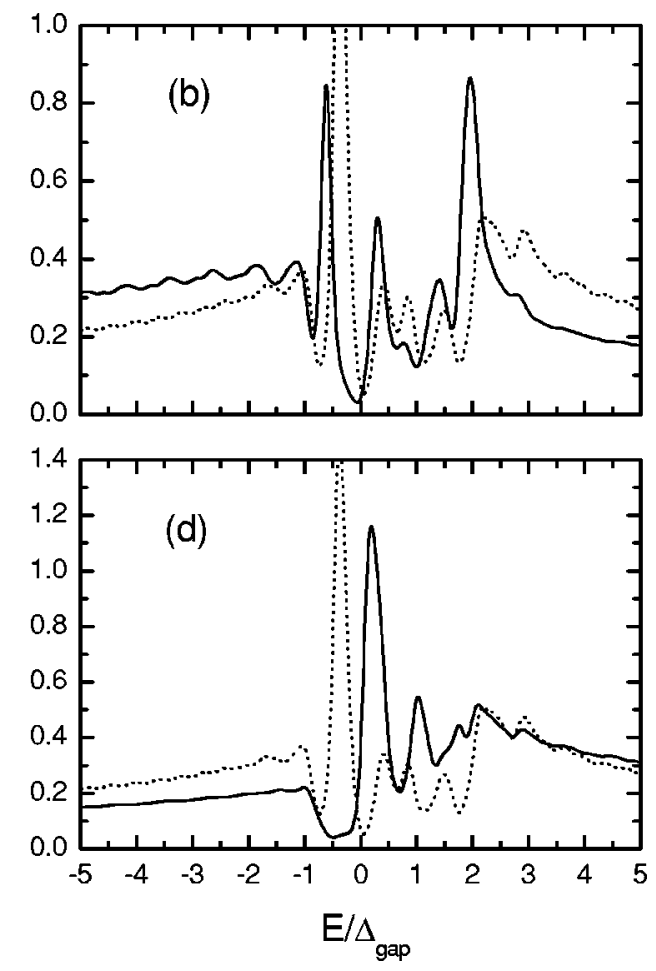

FIG. 4. The LDOS at the impurity site (solid line) in the PS model with $V_{s}=-3$ (a), $V_{s}=-1$ (b), $V_{s}=3$ (c), and $V_{s}=1$ (d). The LDOS at the vortex core without impurity (dotted line) is displayed for direct comparison. 


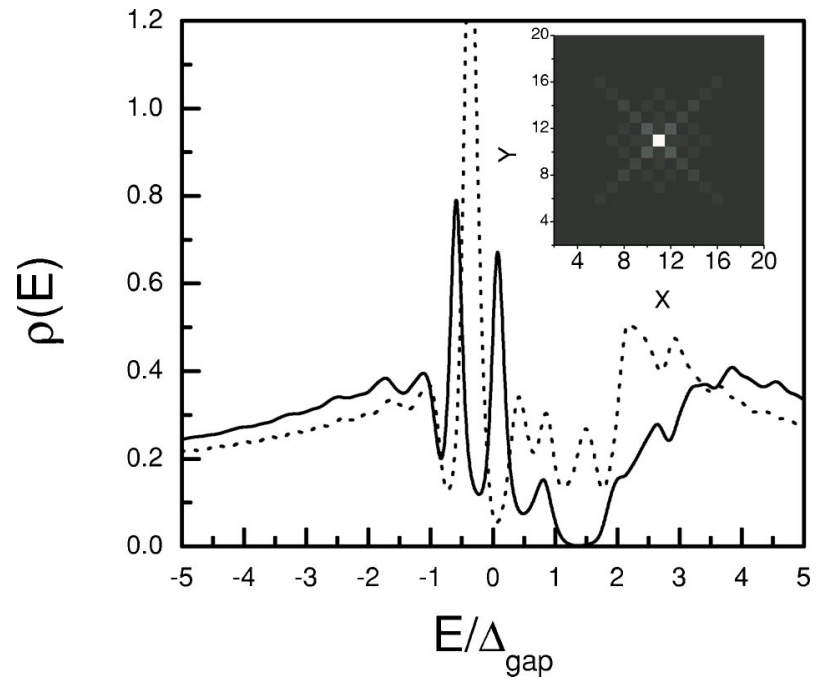

FIG. 5. Same as Fig. 4 except that an Anderson impurity is located at the vortex core. The inset plots the spatial dependence of $\rho(\mathbf{r}, \omega)$ at the lowest-lying resonance energy $\omega / \Delta_{\text {gap }}=0.1$ in one vortex unit cell.

potential $V_{s}=3$, the LDOS in the full energy range displayed in Fig. 4(c) is reduced and we find no impurity state lying within $E_{A B}$ except one at the right side of peak $B$. For $V_{s}=1$, there is a high peak in the vicinity of the Fermi level. As shown in Fig. 4, the scattering potential with the larger magnitude decreases the spectral weight on the impurity site more than that with the smaller one because a large potential tends to exclude the quasiparticle from the scatterer, and in the large limit (unitary) the LDOS right on the impurity site approaches zero. Indeed, we find that for $\left|V_{s}\right| \gg t$, the BdG equations have a simple eigenstate lying far beyond the energy band: the eigenenergy $E \approx V_{s}$ together with the approximate eigenfunctions $u_{i}=\delta_{i, 0}$ and $v_{i}=0$, which indicates that at the impurity site all the spectral weight belonging to the low-energy states will transfer to this high-energy state and thus gives rise to the vanishing of the LDOS of the impurity site within the energy band. Furthermore, unitary impurities (in our study $V_{s}= \pm 100$ ) give rise to impurity resonances infinitely close to the subgap edges, i.e., peaks $A$ and $B$, without any evidence of the near-zero resonant peaks both on the impurity site and around it.

In the AI model, we find that the variation of the magnetization and the electron number around the impurity site is rather small without giving significant modification of the impurity Hamiltonian. For the same parameters as in Fig. 1(b) we find that the net magnetic moment generated around the impurity is rather small with $S_{z}=0.06$, indicating that the impurity spin is strongly screened by the surrounding quasiparticles. For comparison to the PS model, Fig. 5 shows the results of LDOS at the vortex core for the AI model. For the parameters in the strong hybridization regime, a low-energy impurity quasiparticle excitation state is readily obtained and pinned slightly above the Fermi level with $\omega / \Delta_{\text {gap }}=0.1$ in addition to another impurity resonance with a slightly higher energy at the left side of peak $A$. Moreover, comparing with the impurity resonance for the AI model without magnetic field [Fig. 1(b)], we find that (i) the LDOS peak correspond- ing to the impurity state is even strengthened because the impurity resonance is located at the middle of $E_{A B}$ where the residual density of states is low and therefore the intrinsic width of the impurity resonance is narrow; (ii) the impurity state is simply electronlike and has no hole counterpart due to the destruction of the DSC order at the vortex core; (iii) the spatial pattern of the electron part of the quasiparticle excitation is the same as that of the impurity resonance with zero field, as shown in the inset of Fig. 5.

To get a better understanding of the results shown in Figs. 4 and 5 , we also perform a $t$-matrix analysis ${ }^{6,32}$ of the abovementioned results for both models. At the vortex core, the DSC order parameter is so suppressed that the anomalous Green's functions are vanishingly small and for the pointlike scatterers we need only to know the impurity-free normal Green's functions of spin up and down, which are constructed from $E_{n}, u_{n \uparrow}$, and $v_{n \downarrow}$ obtained by solving the BdG equations (4),

$$
\begin{gathered}
G_{\uparrow \uparrow}(0,0, E)=\sum_{n} \frac{\left|u_{n \uparrow}(0,0)\right|^{2}}{E-E_{n}+i \Gamma}, \\
G_{\downarrow \downarrow}(0,0, E)=\sum_{n} \frac{\left|v_{n \downarrow}(0,0)\right|^{2}}{E+E_{n}+i \Gamma},
\end{gathered}
$$

where $\Gamma$ is the broadening parameter with small and positive value. Figure 6 plots the real part of $G_{\uparrow \uparrow}$ and $G_{\downarrow \downarrow}$ as a function of $E$, where we find steep jumps at $E / \Delta_{\text {gap }}=-0.4$ in (a) and 0.4 in (b), corresponding to the $A$ and $B$ peaks in Fig. 2(b). In Fig. 6(a), the energy positions of the marks $A$ and $A^{\prime}$ are slightly different because a small but finite broadening parameter $\Gamma=0.02$ is selected in calculating the Green's functions technically. Theoretically for infinitesimal $\Gamma, E_{A}$ $=E_{A^{\prime}} ; \operatorname{Re} G_{\uparrow \uparrow}(0,0, E)$ should diverge as the energy approaches $E_{A}$ from both sides and the curve connecting $A$ and $A^{\prime}$ will vanish. For Fig. 6(b), we have the same conclusion.

According to the $t$-matrix theory, the existence of impurity resonances in PS models is determined by

$$
\left(\tilde{V}_{s}+\tilde{V}_{m}\right)^{-1}=\operatorname{Re} G_{\uparrow \uparrow}\left(0,0, \omega_{\uparrow}\right)
$$

or

$$
\left(\tilde{V}_{s}-\tilde{V}_{m}\right)^{-1}=\operatorname{Re} G_{\downarrow \downarrow}\left(0,0, \omega_{\downarrow}\right),
$$

where $\omega_{\uparrow}\left(\omega_{\downarrow}\right)$ corresponds to the resonance energy of the impurity state with the spin-up (-down) polarization. Therefore, at energy points where the horizontal lines cross with the line of the real part of the Green's functions as shown in the figure, one finds resonance peaks consistent with the selfconsistent results in Fig. 4. For example, for $V_{s}=-1$, the corresponding horizontal line $\left(\widetilde{V}_{s}+\widetilde{V}_{m}\right)^{-1}$ intersects with the curve of $\operatorname{Re} G_{\uparrow \uparrow}$ at $E<E_{A}$ and thus we get an impurity resonant peak at the left side of peak $A$; similarly an LDOS peak appears rather close to peak $B$ because the horizontal line $\left(\widetilde{V}_{s}-\widetilde{V}_{m}\right)^{-1}$ intersects with $\operatorname{Re} G_{\downarrow \downarrow}$ at the energy $E \lesssim E_{B}$. Moreover, the lines of $\left(\widetilde{V}_{s}+\widetilde{V}_{m}\right)^{-1} \approx\left(\tilde{V}_{s}-\widetilde{V}_{m}\right)^{-1} \approx 0$ corresponding to the unitary impurity do not cut the curves of the 

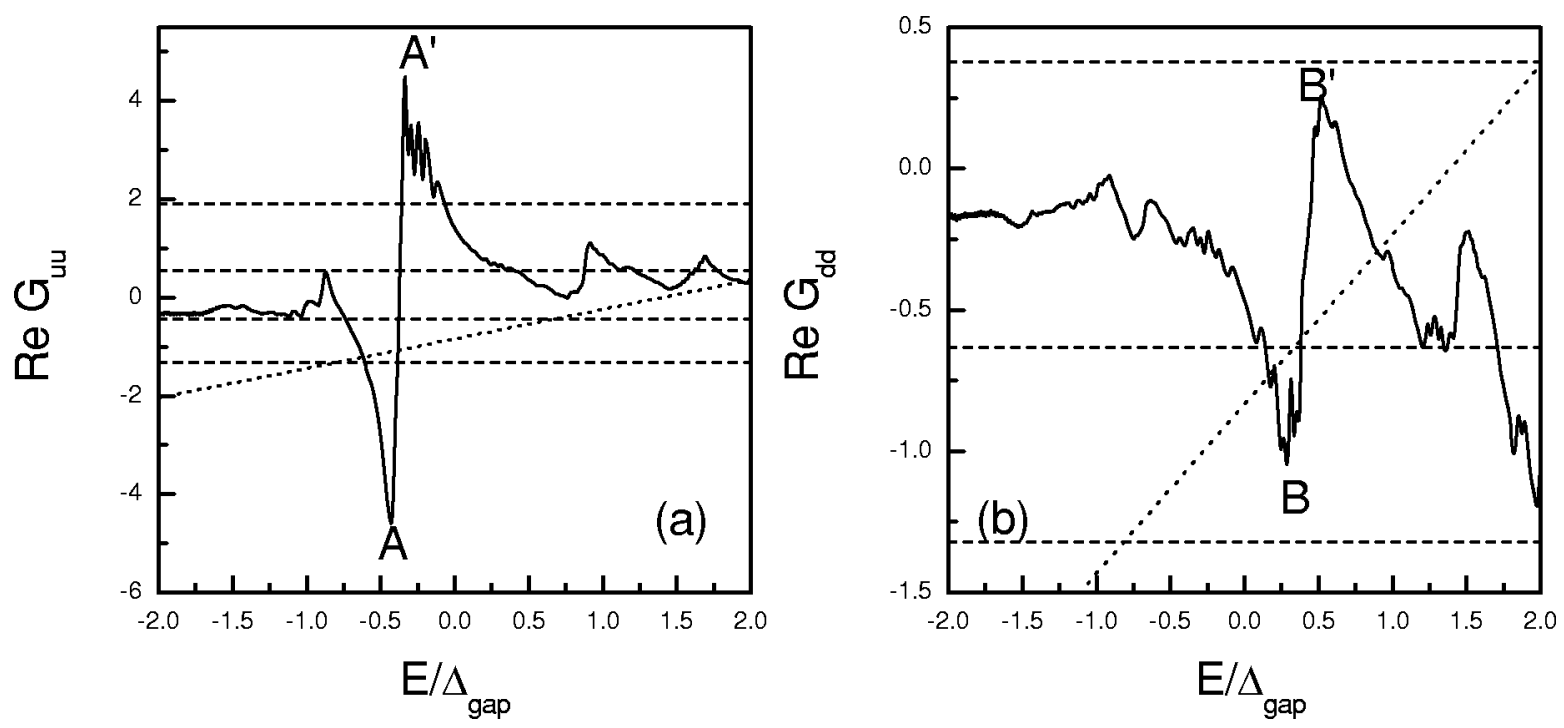

FIG. 6. Real parts of the $G_{\uparrow \uparrow}$ (a) and $G_{\downarrow \downarrow}$ (b) at the vortex core as a function of energy are shown by the solid lines. Dashed lines from bottom to top correspond to $\left(\widetilde{V}_{s}+\widetilde{V}_{m}\right)^{-1}=-1.32,-0.42,0.56,1.92$ in (a) and $\left(\widetilde{V}_{s}-\widetilde{V}_{m}\right)^{-1}=-1.32,-0.63,0.38,1.09$ in (b) with $V_{s}=-1$, $-3,3,1$. Displayed in the dotted slope line is the $V_{\mathrm{eff}}^{-1}(E)=\left(E-\widetilde{\epsilon}_{d}\right)\left(\widetilde{t}_{h}\right)^{-2}$. The broadening parameter $\Gamma=0.02$.

Green's functions in the subgap region, so there is no lowenergy impurity resonance.

On the other hand, for the AI model, after we integrate out the impurity degree of freedom, the conduction electron at the impurity site will feel an energy-dependent pointlike scattering potential with $V_{\text {eff }}^{-1}(E)=\left(E-\widetilde{\epsilon}_{d}\right)\left(\widetilde{t}_{h}\right)^{-2}$, where $\tilde{\epsilon}_{d}$ $\approx 0.5$ and $\tilde{t}_{h} \approx 0.82$ derived from calculation of the minimum of the free energy. Then in the AI model, the equations determining the impurity resonant energies will be

$$
V_{\text {eff }}^{-1}\left(\omega_{\uparrow}\right)=\operatorname{Re} G_{\uparrow \uparrow}\left(0,0, \omega_{\uparrow}\right)
$$

or

$$
V_{\text {eff }}^{-1}\left(\omega_{\downarrow}\right)=\operatorname{Re} G_{\downarrow \downarrow}\left(0,0, \omega_{\downarrow}\right) .
$$

$V_{\text {eff }}^{-1}(E)$ is plotted as the dotted line in Fig. 6. Because $V_{\text {eff }}^{-1}(E)$ crosses $\operatorname{Re} G_{\uparrow \uparrow}$ at the energy point which is very close to that of the PS model with $V_{s}=-1$, the LDOS peak at the left side of peak $A$ in Fig. 5 is similar to that in Fig. 4(b). And the fact that it intersects with $\operatorname{Re} G_{\downarrow \downarrow}$ in the subgap $E_{A B}$ gives rises to the low-lying impurity resonance as shown in Fig. 5.

From Fig. 6, we find that both the PS model and the AI model are able to produce the low-lying impurity resonance for certain parameters. However, to achieve a clear-cut impurity state which separates with the vortex core states apparently, the resonance energy should lie within a region smaller than the subgap $E_{A B}$. We find that the impurity states in the PS model have shown a behavior which is sensitively dependent on the scattering potential. Nevertheless, for the AI model as long as the strength of hybridization between the $d$ electron and conduction electrons is strong enough, $t_{h}$ $\gtrsim 1$, there is always an impurity resonance pinned near the Fermi level according to our numerical calculations. Moreover, the lowest-lying impurity state (nearest to the Fermi level) of the AI model and negative PS model has opposite spin polarization to the magnetization of the vortex core, while that of the positive PS model has the same polarization as the magnetization of the core.

Specifically, for the Niimpurity our numerical calculation indicates that in the PS model $\left(V_{s}=-3\right)$ the impurity state in the vortex core has a resonance energy quite close to that of the vortex state $E_{A}$, while in the AI model $\left(t_{h}=1\right)$ the impurity resonance energy is close to zero, resulting in an LDOS peak much sharper than that of the PS model. Future STM observation of the Niimpurity in the magnetic field will shed light on the dominant mechanism of the impurity resonant state in the HTS.

\section{SUMMARY AND DISCUSSION}

In this work, we have compared the potential scattering model with the Anderson model by examining the quasiparticle excitation around the $\mathrm{Ni}$ impurity doped in $d$-wave superconductors. At zero field, both the PS model and the AI model can produce the impurity resonant state with the resonant energy and spatial pattern qualitatively consistent with one of the two differential conductance peaks within the superconducting gap observed around the $\mathrm{Ni}$ atom doped in HTS by the STM experiment. After applying an external magnetic field larger than the lower critical field, the impurity states generated by both models are quite different from those in zero field. In the AI model, the impurity state is pinned within the subgap opened by the static AF order at the vortex core with extreme particle-hole asymmetry. Furthermore, the impurity state has a spin polarization contrary to that of the AF order of the vortex core, while in the PS model, both the resonant energy and the spin polarization of the lowest-lying resonant state are rather sensitive to the sign and strength of the scattering potential. We propose highresolution spin-polarized STM experiments to probe not only the spin structure of the vortex core but also the existence and polarization of possible low-lying impurity quasiparticle 
excitations when vortices are pinned by the doped impurity atom such as $\mathrm{Ni}$ in the $\mathrm{CuO}_{2}$ planes of HTS.

From the impurity Hamiltonian, the remarkable difference between the PS and AI models is that the AI model introduces an additional degree of freedom to describe the magnetic momenta carried/induced by the impurity site. In the framework of the Green's function theory, this extra degree of freedom can be integrated out, which results in the AI impurity acting like a scalar potential scatterer. However, the effective potential corresponding to the AI impurity is energy-dependent, which makes the PS model and AI model essentially different because in the PS model the scattering potential is energy-independent. In this paper, by depositing the impurity in the insulating vortex core with a small gap opened by the staggered magnetization, which plays a key role in the suppression of the superconducting order in the vortex core for both cases and is consistent with the STM observation, ${ }^{23}$ we have displayed such differences between the PS and AI models. Note that, even if the static AF order vanishes and the vortex core becomes metallic, the impurity states of the PS and AI models are still different, which agrees with what we found in an earlier work. ${ }^{13}$

Finally, we wish to discuss also the impurity state of $\mathrm{Zn}$ briefly, when a vortex line is pinned on it with the induced static AF order. Similar to the case of Ni studied above, in zero field both the PS model in the unitary limit ${ }^{6,8}$ and the AI model $^{12,13}$ [or alternative models taking into account the effect of the magnetic moment induced around Zn (Ref. 10)] can give rise to the impurity resonance, explaining qualitatively the experiment data. ${ }^{2}$ However, in the presence of the flux line on the impurity site, we obtain no low-lying impurity state within the small subgap opened by the static AF order for the large (negative and positive) scattering potential, while for the AI model for $\mathrm{Zn}$ with the four-site hybridization proposed in Refs. 12 and 13, the impurity resonant state with near zero energy is seen. ${ }^{33}$ Therefore, to examine whether or not the low-lying impurity excitation exists when the magnetic field is applied in the $\mathrm{Zn}$-doped superconducting material may help us to elucidate the dominant mechanism of the impurity resonance.

\section{ACKNOWLEDGMENTS}

We thank Q.-H. Wang for helpful discussions. The work was supported by the RGC grant of Hong Kong under Grant Nos. HKU7092/01P and HKU7075/03P, the 973-project of the Ministry of Science and Technology of China under Grant Nos. G1999064602 and G1999064603, the URC fund of HKU, and the National Natural Science Foundation of China under Grant Nos. 10204019 and 10334090.
*Electronic address: zwang@hkucc.hku.hk

${ }^{1}$ E. W. Hudson, S. H. Pan, A. K. Gupta, K.-W. Ong, and J. C. Davis, Science 285, 88 (1999); A. Yazdani, C. M. Howald, C. P. Lutz, A. Kapitulnik, and D. M. Eigler, Phys. Rev. Lett. 83, 176 (1999); N.-C. Yeh, C.-T. Chen, G. Hammerl, J. Mannhart, A. Schmehl, C. W. Schneider, R. R. Schulz, S. Tajima, K. Yoshida, D. Garrigus, and M. Strasik, ibid. 87, 087003 (2001).

${ }^{2}$ S. H. Pan, E. W. Hudson, K. M. Lang, H. Eisaki, S. Uchida, and J. C. Davis, Nature (London) 403, 746 (2000).

${ }^{3}$ E. W. Hudson, K. M. Lang, V. Madhavan, S. H. Pan, H. Eisaki, S. Uchida, and J. C. Davis, Nature (London) 411, 920 (2001).

${ }^{4}$ J. Bobroff, W. A. MacFarlane, H. Alloul, P. Mendels, N. Blanchard, G. Collin, and J.-F. Marucco, Phys. Rev. Lett. 83, 4381 (1999); M.-H. Julien, T. Fehér, M. Horvatić, C. Berthier, O. N. Bakharev, P. Ségransan, G. Collin, and J.-F. Marucco, ibid. 84, 3422 (2000); J. Bobroff, H. Alloul, W. A. MacFarlane, P. Mendels, N. Blanchard, G. Collin, and J.-F. Marucco, ibid. 86, 4116 (2001).

${ }^{5}$ H. F. Fong, P. Bourges, Y. Sidis, L. P. Regnault, J. Bossy, A. Ivanov, D. L. Milius, I. A. Aksay, and B. Keimer, Phys. Rev. Lett. 82, 1939 (1999); Y. Sidis, P. Bourges, H. F. Fong, B. Keimer, L. P. Regnault, J. Bossy, A. Ivanov, B. Hennion, P. Gautier-Picard, G. Collin, D. L. Millius, and I. A. Aksay, ibid. 84, 5900 (2000).

${ }^{6}$ A. V. Balatsky, M. I. Salkola, and A. Rosengren, Phys. Rev. B 51, 15547 (1995); M. I. Salkola, A. V. Balatsky, and D. J. Scalapino, Phys. Rev. Lett. 77, 1841 (1996); M. I. Salkola, A. V. Balatsky, and J. R. Schrieffer, Phys. Rev. B 55, 12648 (1997); I. Martin, A. V. Balatsky, and J. Zaanen, Phys. Rev. Lett. 88, 097003 (2002).
${ }^{7}$ M. E. Flatte and J. M. Byers, Phys. Rev. B 56, 11213 (1997); M. E. Flatte and J. M. Byers, Phys. Rev. Lett. 80, 4546 (1998); J. Tang and M. E. Flatte, Phys. Rev. B 66, 060504 (2002).

${ }^{8}$ J. X. Zhu, C. S. Ting, and C.-R. Hu, Phys. Rev. B 62, 6027 (2000); J. X. Zhu, T. K. Lee, C. S. Ting, and C.-R. Hu, ibid. 61, 8667 (2000).

${ }^{9}$ H. Tsuchiura, Y. Tanaka, M. Ogata, and S. Kashiwaya, J. Phys. Soc. Jpn. 68, 2510 (1999); Phys. Rev. Lett. 84, 3165 (2000).

${ }^{10}$ A. Polkovnikov, S. Sachdev, and M. Vojta, Phys. Rev. Lett. 86, 296 (2000); A. Polkovnikov, Phys. Rev. B 65, 064503 (2002); M. Vojta and R. Bulla, ibid. 65, 014511 (2002).

${ }^{11}$ J.-X. Zhu and C. S. Ting, Phys. Rev. B 63, 020506(R) (2001); 64, 060501(R) (2001).

${ }^{12}$ G. M. Zhang, H. Hu, and L. Yu, Phys. Rev. Lett. 86, 704 (2001); Phys. Rev. B 66, 104511 (2002).

${ }^{13}$ Q. Han, Z. D. Wang, X.-G. Li, and L.-y. Zhang, Phys. Rev. B 66, 104502 (2002).

${ }^{14}$ In this work, we concentrate on the response of the impurity resonance to the presence of $\mathrm{AF}$ order at the vortex core and therefore we study the single-peak impurity state case for simplicity. To fully explain the two LDOS peaks for each Ni atom observed by the STM experiment, one can simply choose the PS model with $V_{m} \neq 0$ or the AI model, which also considers the effect of the scattering potential of the Anderson impurity.

${ }^{15}$ Y. Wang and A. H. MacDonald, Phys. Rev. B 52, R3876 (1995).

${ }^{16}$ D. P. Arovas, A. J. Berlinsky, C. Kallin, and S.-C. Zhang, Phys. Rev. Lett. 79, 2871 (1997); J. H. Han and D. H. Lee, ibid. 85, 1100 (2000); Y. Zhang, E. Demler, and S. Sachdev, Phys. Rev. B 66, 094501 (2001).

${ }^{17}$ J.-X. Zhu and C. S. Ting, Phys. Rev. Lett. 87, 147002 (2001); 
J.-X. Zhu, I. Martin, and A. R. Bishop, ibid. 89, 067003 (2002).

${ }^{18}$ Y. Chen and C. S. Ting, Phys. Rev. B 65, 180513(R) (2002); Y. Chen, H. Y. Chen, and C. S. Ting, ibid. 66, 104501 (2002); Y. Chen, Z. D. Wang, J.-X. Zhu and C. S. Ting, Phys. Rev. Lett. 89, 217001 (2002); Y. Chen, Z. D. Wang, and C. S. Ting, Phys. Rev. B 67, 220511 (2003).

${ }^{19}$ A. Ghosal, C. Kallin, and A. J. Berlinsky, Phys. Rev. B 66, 214502 (2002).

${ }^{20}$ M. Takigawa, M. Ichioka, and K. Machida, Phys. Rev. Lett. 90, 047001 (2003).

${ }^{21}$ J. Kishine, P. A. Lee, and X.-G. Wen, Phys. Rev. Lett. 86, 5365 (2001)

${ }^{22}$ Q.-H. Wang, J. H. Han, and D.-H. Lee, Phys. Rev. Lett. 87, 167004 (2001).

${ }^{23}$ I. Maggio-Aprile, Ch. Renner, A. Erb, E. Walker, and Ø. Fischer, Phys. Rev. Lett. 75, 2754 (1995); Ch. Renner, B. Revaz, K. Kadowaki, I. Maggio-Aprile, and Ø. Fischer, ibid. 80, 3606 (1998); S. H. Pan, E. W. Hudson, A. K. Gupta, K.-W. Ng, H. Eisaki, S. Uchida, and J. C. Davis, ibid. 85, 1536 (2000).

${ }^{24}$ B. Lake, G. Aeppli, K. N. Clausen, D. F. McMorrow, K. Lefmann, N. E. Hussey, N. Mangkorntong, M. Nohara, H. Takagi, T. E. Mason, and A. Schröder, Science 291, 1759 (2001); B. Lake, H. M. Rønnow, N. B. Christensen, G. Aeppli, K. Lefmann, D. F. McMorrow, P. Vorderwisch, P. Smeibidl, N. Mangkorntong, T. Sasagawa, M. Nohara, H. Takagi, and T. E. Mason, Nature (London) 415, 299 (2002).

${ }^{25}$ V. F. Mitrović, E. E. Sigmund, M. Eschrig, H. N. Bachman, W. P. Halperin, A. P. Reyes, P. Kuhns, and W. G. Moulton, Nature (London) 413, 501 (2001); V. F. Mitrović, E. E. Sigmund, W. P. Halperin, A. P. Reyes, P. Kuhns, and W. G. Moulton, cond-mat/ 0202368 (unpublished).

${ }^{26}$ K. Kakuyanagi, K. Kumagai, Y. Matsuda, and M. Hasegawa, Phys. Rev. Lett. 90, 197003 (2003).
${ }^{27}$ S. E. Barnes, J. Phys. F: Met. Phys. 6, 1375 (1976); P. Coleman, Phys. Rev. B 29, 3035 (1984).

${ }^{28}$ As shown in Fig. 1 (dotted lines), coherence peaks are located at $E / \Delta_{\text {gap }}= \pm 1$. At the vicinity of the coherence peaks (especially above $E / \Delta_{\text {gap }}=1$ and below the peak of the van Hove singularity with $E / \Delta_{\text {gap }}=1.5$ ), there are also peaks which arise from the splitting effect of the AFM order induced by the impurity. Due to the finite size, the AFM order induced by the impurity still has nonzero value on the subsystem boundary, which lifts the spin up-down degeneracy and results in the splitting. Furthermore, because the magnetization is vanishingly small on the impurity site itself (with maximum magnitude on the $\mathrm{NN}$ sites), the $(0,0)$ LDOS peak corresponding to the impurity resonance state is not split as indicated by Fig. 1.

${ }^{29}$ Q. Han, Z. D. Wang, L.-y. Zhang, and X.-G. Li, Phys. Rev. B 65, 064527 (2002).

${ }^{30}$ Y. Chen and C. S. Ting, Phys. Rev. Lett. 92, 077203 (2004).

${ }^{31}$ In the identification of the LDOS peaks of the impurity states from those of the vortex core states, we apply the $t$-matrix theory addressed below. According to this theory, $\omega_{\uparrow}$ or $\omega_{\downarrow}$ satisfying Eqs. (14) and (15) for the PS model and Eqs. (16) and (17) for the AI model denotes the resonance energy of the impurity state with spin polarization up or down. Here we will concentrate on the low-lying impurity states in the vicinity of the Fermi level within $E_{A B}$, because for $E$ in this range the imaginary part of $G_{\uparrow \uparrow}(0,0, E)$ and $G_{\downarrow \downarrow}(0,0, E)$ is vanishingly small [consequently the LDOS at the impurity site in $E_{A B}$ is small, as shown in Fig. 2(b)] , and therefore the intrinsic width of the low-lying impurity states will be small and will likely be observed by STM observation.

${ }^{32}$ Q.-H. Wang and Z. D. Wang, Phys. Rev. B 69, 092502 (2004).

${ }^{33} \mathrm{Q}$. Han et al. (unpublished). 